\title{
Women's intention to screen and willingness to vaccinate their daughters against cervical cancer - a cross sectional study in eastern Uganda
}

Rawlance Ndejjo (D), Trasias Mukama, Geofrey Musinguzi, Abdullah Ali Halage, John C. Ssempebwa and David Musoke

\begin{abstract}
Background: The World Health Organization recommends cervical cancer screening and vaccination programmes as measures to combat cervical cancer. The uptake of these measures remains low in Uganda, most especially in rural areas. An understanding of the factors that influence women's decision to attend screening, and willingness to have their daughters vaccinated against cervical cancer is essential for any attempts to increase uptake of these services. This study assessed the factors associated with intention to screen for cervical cancer among women in eastern Uganda, and willingness to have their daughters vaccinated against the disease.

Methods: This cross sectional study involved 900 females aged 25 to 49 years in Bugiri and Mayuge districts in eastern Uganda. Data were collected using a pretested semi-structured questionnaire, entered in Epidata version 3.02 and analysed in STATA version 12.0. Unadjusted and adjusted prevalence ratios (PR) were computed using a generalized linear model with Poisson family, and a log link with robust standard errors.

Results: Majority 819 (91.0\%) of respondents stated that they intended to go for cervical cancer screening in the subsequent six months. Among them, 603 (73.6\%) wanted to know their status, 256 (31.3\%) thought it was important, 202 (24.7\%) wanted to reduce their chances of getting the disease, and 20 (2.4\%) had been told to do so by a health worker. Majority $813(90.4 \%)$ of respondents were willing to vaccinate their daughters against cervical cancer. Higher income (adjusted PR $=1.11,95 \% \mathrm{Cl}: 1.03-1.20$ ), cervical cancer screening status (adjusted PR $=0.81,95 \% \mathrm{Cl}: 0.67-0.99$ ) and knowledge of at least one test for cervical cancer (adjusted PR $=0$. 92, 95\% Cl: 0.85-0.98) were significantly associated with intention to screen for cervical cancer. No socio-demographic characteristic was associated with willingness to vaccinate daughters among women.
\end{abstract}

Conclusion: There is a very high intention to screen and willingness to vaccinate daughters against cervical cancer among women in eastern Uganda. To take advantage of this, there is need to avail opportunities for women to access cervical cancer screening and vaccinations particularly among rural communities.

Keywords: Cervical cancer, Intention to screen, Rural, Screening, Uganda, Vaccination, Willingness

\footnotetext{
* Correspondence: rndejjo@musph.ac.ug

Department of Disease Control and Environmental Health, School of Public

Health, College of Health Sciences, Makerere University, Kampala, Uganda
} 


\section{Background}

Cervical cancer remains a major cause of morbidity and mortality among women in resource constrained settings due to low access to cancer screening and vaccination $[1,2]$. Indeed, coverage of cancer screening services is lowest in low and middle income countries [3]. These countries have only a few trained and skilled health workers to carry out screening, and lack healthcare resources to sustain such programmes [4]. Consequently, women with cervical cancer are not identified until they are at an advanced stage of disease, resulting in high morbidity and mortality [4]. On the other hand, many developing countries are still in the initial stages of rolling out cervical cancer vaccination among their populations. For example, in Uganda, cervical cancer vaccinations among girls aged between 9 to 13 years in schools and the community were piloted in 2008 and a national strategic plan launched in 2010 [5, 6]. Other sub-Saharan African countries such as Ghana and Kenya have also rolled out cervical cancer vaccinations among their population $[7,8]$.

Cervical cancer is potentially preventable, and effective screening programmes can lead to a significant reduction in the morbidity and mortality associated with this cancer [2, 9] especially if detected in early stages [10]. The World Health Organization (WHO) recommends screening and vaccination programmes globally [11]. However, only a small percentage $(5-27 \%)$ of women in sub-Saharan Africa report having received cervical cancer screening [12-19]. This is even lower in the East African region where the prevalence of human papilloma virus (HPV) (20\%) is high, and the incidence rates of cervical cancer are highest [20]. Understanding factors that influence intention to screen for cervical cancer among women is important to inform programmes aimed at increasing utilisation of screening services in sub-Saharan Africa. Previous studies show that women's intention to screen, and attendance of cervical cancer screening is influenced by: demographic factors such as occupation, living with a partner; attitudinal factors such as perception of risk and disease; social factors for example discussions by health workers; and health system factors such as distance to health facilities and awareness of the service [18, 19].

HPV, which is mainly sexually transmitted, causes most of the cervical cancer cases [21]. Highly effective prophylactic vaccines against HPV -16 and HPV -18 have been shown to prevent over $70 \%$ of these cases worldwide [22]. HPV vaccinations can therefore significantly reduce the incidence of cervical cancer among women. Moreover, WHO recommends offering HPV vaccine to girls between 9 and 13 years, prior to sexual debut, as the vaccine is most efficacious if girls have not already been exposed to the virus [23]. The success of the vaccine would depend on levels of acceptability and uptake, which heavily relies on parent's willingness to have their eligible daughters vaccinated [24]. Prior research among parents has reported predictors of vaccinations of girls to include perception of the severity of the disease, belief of daughter being at risk, higher education and income, attitudes towards vaccines, previous awareness of the vaccine, and recommendations by vaccine providers [7, 25-28]. Barriers to cervical cancer vaccination such as concerns about vaccine safety, danger to daughter, sexual behaviours, and lack of provider recommendation have also been highlighted [7, 25-29]. However, there has been a paucity of such studies in Uganda to provide context specific barriers and inform on-going vaccine promotion campaigns. This study assessed the factors associated with intention to screen for cervical cancer among women in eastern Uganda, and willingness to have their daughters vaccinated against the disease.

\section{Methods \\ Study area}

The study was conducted in Bugiri and Mayuge districts in eastern Uganda approximately 150 kilometres from Kampala, the country's capital city. These predominantly rural districts have a combined area of $10,372 \mathrm{~km}^{2}$ and a total population of 856,152 people of whom $51.4 \%$ are females [30]. Bugiri district is composed of 9 subcounties while Mayuge has 7 sub-counties. Majority of people in the districts reside in roofed mud and wattle houses. Residents majorly participate in agriculture for subsistence though a few operate small businesses in trading centres, and others engage in fishing along the shores of Lake Victoria. In the two districts, cervical cancer screening is provided by Bugiri district hospital, a government health facility that also serves neighbouring districts. The hospital provides intermittent screening services and treatment of those diagnosed with the disease. Three (3) private health facilities, 2 in Bugiri and 1 in Mayuge district, also provide cervical cancer screening.

\section{Study design and population}

This was a cross sectional study that employed quantitative data collection methods. The study involved females aged 25 to 49 years in Bugiri and Mayuge districts who had lived in the area for at least six months. A minimum sample size of 845 was determined using the sample size estimation formula for cross sectional studies [31] with $\mathrm{Z}=1.96, p=0.5$ (used to obtain the maximum sample size since proportion was not known), and a precision of $5 \%$ taking into account a design effect of 2.0 , and a nonresponse rate of $10 \%$. The sampling units were households, and only one participant was interviewed per 
selected household with priority given to household heads or their spouses.

\section{Sampling}

Multi-stage sampling technique was used as follows: five sub-counties were randomly selected from each district. Five villages were then selected from each sub-county using simple random sampling to obtain 25 study villages in each district. Lists of sub-counties and villages were obtained from district officials while village Local Council leaders provided estimates of numbers of households in their villages. The study households were selected using systematic random sampling where the interval for selection was determined by dividing the approximate number of households in a given village by the required number of respondents.

\section{Data collection}

Data were collected using a pretested semi-structured questionnaire that was developed in English, and then translated to Lusoga, the main local language of the area. The questionnaire had questions on knowledge and attitudes towards cervical cancer and screening, access to cervical cancer screening and intention to screen, respondent willingness to vaccinate their daughters, and socio-demographic factors whose details have been described elsewhere [19]. The questionnaire was administered by a team of trained Research Assistants at respondents' homes.

\section{Data analysis}

Data were entered in Epidata version 3.02 (EpiData Association, Denmark) and analysed in STATA version 12.0 (Stata Corp, Texas, USA) statistical software. The outcome variables were intention to screen for cervical cancer in the following six months, and willingness for vaccination of daughters against cervical cancer. Intention to screen was assessed using a question which asked respondents "Do you intend to go for cervical cancer screening in the next six months?" with three responses of 'Yes' 'No' and "Undecided". 'Yes' and 'No' responses formed the binary outcome variable and were assigned 1 and 0 respectively during further analysis. For willingness to vaccinate daughters against cervical cancer, respondents answered to the statement "I would allow my children to be vaccinated against cervical cancer" with response based on a 5 point - Likert scale ranging from strongly disagree to strongly agree. Respondents who gave 'Strongly agree' and 'Agree' responses were coded as 'willing to allow their daughters receive vaccination' and assigned 1 while those who responded with 'Strongly disagree' and 'Disagree' responses were coded as not willing to allow their daughters to be vaccinated' (assigned 0 ). Those who were not sure were dropped in creating this outcome variable. Prevalence ratios (PR) computed using a generalized linear model with Poisson family, and a log link with robust standard errors while applying a backward elimination method were used to measure the association between the outcome and independent variables. PRs were used because the outcomes were highly prevalent $(>10 \%)$ as odds ratios tend to overestimate the relative risk in such instances [32-34]. Simple models were run to obtain the unadjusted PRs, and then basing on biological plausibility and $p<0.05$, variables from these models were included in the multivariable model. We present both the unadjusted and adjusted PRs, and their corresponding 95\% confidence intervals $(\mathrm{CI})$ as well as p-values. A p-value of less than 0.05 was considered for a statistically significant association.

\section{Results}

Socio-demographic characteristics of respondents

All the 900 respondents selected took part in the study. Majority of these were from rural areas $610(67.8 \%)$ and married $767(85.2 \%)$. Only a quarter $228(25.3 \%)$ of respondents had attained post primary education (Table 1).

\section{Intention to screen for cervical cancer}

Majority $819(91.0 \%)$ of respondents stated that they intended to go for cervical cancer screening in the following six months. Only 57 (6.3\%) did not intend to undergo the procedure while $24(2.7 \%)$ were undecided. Reasons for intention to screen were: $603(73.6 \%)$ to know status, 256 (31.3\%) thought it was important, 202 (24.7\%) wanted to reduce their chances of getting the disease, and $20(2.4 \%)$ had been told to do so by a health worker. Among those who did not intend to screen, 28 (49.1\%) stated that it was not necessary or important, 9 $(15.8 \%)$ cited health facility being far away, $6(10.5 \%)$ high costs involved, 5 (8.8\%) said they had been screened recently, and 13 (22.8\%) gave other reasons (fear of pain, lack of signs and symptoms, and already undergoing treatment). Among respondents who had never screened for cervical cancer, intention to screen was at $91.6 \%$ (785/857). When respondents were asked whether they would be willing to undergo screening if the service was offered at an affordable cost to them, majority 828 (92.0\%) said they would. Moreover, 863 (95.9\%) stated willingness to screen if the service was provided free of charge.

\section{Respondents' willingness for vaccination of themselves and their daughters}

Of the study respondents, more than nine in ten 815 (90.5\%) reported willingness to be vaccinated against cervical cancer if it were recommended. The rest reported unwillingness $61(6.8 \%)$ or uncertainty $24(2.7 \%)$. 
Table 1 Socio-demographic characteristics of respondents $(N=900)$

\begin{tabular}{|c|c|c|}
\hline Characteristic & Categories & n (\%) \\
\hline \multirow[t]{2}{*}{ District } & Bugiri & $452(50.2)$ \\
\hline & Mayuge & $448(49.8)$ \\
\hline \multirow[t]{2}{*}{ Residence } & Rural & $610(67.8)$ \\
\hline & Semi-urban/urban & $290(32.2)$ \\
\hline \multirow[t]{3}{*}{ Age (years) } & Mean (SD) & $32.9( \pm 6.7)$ \\
\hline & $25-39$ & $703(78.1)$ \\
\hline & $40-49$ & $197(21.9)$ \\
\hline \multirow[t]{2}{*}{ Education level } & None/primary & $672(74.7)$ \\
\hline & Post primary & $228(25.3)$ \\
\hline \multirow[t]{2}{*}{ Religion } & Muslims & $382(42.4)$ \\
\hline & Christians & $518(57.6)$ \\
\hline \multirow[t]{2}{*}{ Marital status } & Single & $133(14.8)$ \\
\hline & Married & $767(85.2)$ \\
\hline \multirow[t]{2}{*}{ Occupation } & Farming & $502(55.8)$ \\
\hline & $\begin{array}{l}\text { Others (business, } \\
\text { housewife, civil servant) }\end{array}$ & $195(21.7)$ \\
\hline \multirow[t]{3}{*}{ Parity } & Mean (SD) & $5.04( \pm 2.7)$ \\
\hline & Four and below & $430(47.8)$ \\
\hline & Above four & $470(52.2)$ \\
\hline \multirow[t]{2}{*}{ Household monthly income } & Less than $\$ 40$ & $622(69.1)$ \\
\hline & $\$ 40$ and above & $278(30.9)$ \\
\hline \multirow[t]{2}{*}{ Household head } & No & $757(84.1)$ \\
\hline & Yes & $143(15.9)$ \\
\hline \multirow[t]{2}{*}{ Ever tested for HIV } & No & $144(16.0)$ \\
\hline & Yes & $756(84.0)$ \\
\hline \multirow{2}{*}{$\begin{array}{l}\text { Ever used modern family } \\
\text { planning method }\end{array}$} & No & $317(35.2)$ \\
\hline & Yes & $583(64.8)$ \\
\hline \multirow[t]{2}{*}{ Cervical cancer screening status } & Not screened & $857(95.2)$ \\
\hline & Screened & $43(4.8)$ \\
\hline
\end{tabular}

Majority 813 (90.4\%) also reported that they would allow their daughters to be vaccinated against cervical cancer, $59(6.6 \%)$ wouldn't and the rest 28 (3.1\%) were undecided.

\section{Socio-demographic characteristics associated with intention to screen, and willingness to vaccinate daughters against cervical cancer}

Respondents from Mayuge district (unadjusted $P R=1.10$, 95\% CI $=1.06-1.14)$ and those whose households earned 40 US dollars or more per month (unadjusted $\mathrm{PR}=1.07$, $95 \% \mathrm{CI}=1.04-1.11$ ) had a $10 \%$ and $7 \%$ higher intention to screen for cervical cancer respectively compared with their counterparts. On the other hand, respondents who had accessed cervical cancer screening were less likely to intend to undergo another test in the subsequent six months (unadjusted $\mathrm{PR}=0.86,95 \% \mathrm{CI}=0.74-0.99$ ). The respondents' district of residence (unadjusted $\mathrm{PR}=1.05$, $95 \% \mathrm{CI}=1.01-1.09$ ), residence in a rural or urban area (unadjusted $\mathrm{PR}=1.05,95 \% \mathrm{CI}=1.01-1.08$ ), occupation (unadjusted $\mathrm{PR}=0.94,95 \% \mathrm{CI}=0.91-0.98$ ), and household income (unadjusted PR $=1.05,95 \% \mathrm{CI}=1.01$ 1.08) were all significantly associated with willingness to vaccinate their daughters against cervical cancer (Table 2).

\section{Knowledge factors associated with intention to screen,} and willingness to vaccinate daughters against cervical cancer

Reporting that early detection of cervical cancer is helpful (unadjusted $\mathrm{PR}=1.18,95 \% \mathrm{CI}=1.02-1.36$ ), knowing that one could be vaccinated against cervical cancer (unadjusted $\mathrm{PR}=1.05,95 \% \mathrm{CI}=1.01-1.09$ ), and knowledge of at least one test for cervical cancer (unadjusted $\mathrm{PR}=0.95,95 \% \mathrm{CI}=0.91-0.98)$ were significantly associated with intention to screen for cervical cancer at bivariate analysis. On the other hand, knowing that one could be vaccinated against cervical cancer (unadjusted $\mathrm{PR}=1.05,95 \% \mathrm{CI}=1.01-1.10)$, knowing more than one preventive measure (unadjusted $\mathrm{PR}=0.96,95 \% \mathrm{CI}=0.91$ 0.99 ) or symptom of cervical cancer (unadjusted $\mathrm{PR}=$ 0.94, 95\% CI $=0.01-0.97$ ), and knowledge of at least one test for cervical cancer (unadjusted $\mathrm{PR}=0.96,95 \% \mathrm{CI}=$ $0.93-0.99$ ) were significantly associated with willingness to vaccinate daughters against cervical cancer (Table 3 ).

\section{Attitude factors associated with intention to screen and} willingness to vaccinate daughters against cervical cancer Majority 796 (96.1\%) of respondents thought that cervical cancer was a severe disease and these had a significantly higher intention to screen (unadjusted $P R=2.12$, $95 \% \mathrm{CI}=1.52-2.96)$. Other attitudinal factors associated with intention to screen for cervical cancer were: belief of being at risk of getting cervical cancer (unadjusted $\mathrm{PR}=1.29,95 \% \mathrm{CI}=1.14-1.48$ ), stating that cervical cancer screening is important (unadjusted $\mathrm{PR}=1.81,95 \%$ $\mathrm{CI}=1.34-2.45$ ), and that chances of curing cervical cancer are better when it is discovered at an early stage (unadjusted $\mathrm{PR}=1.22,95 \% \mathrm{CI}=1.11-1.34$ ), and that their family would approve of children being vaccinated against cervical cancer (unadjusted $\mathrm{PR}=1.70,95 \% \mathrm{CI}=$ 1.36-2.13). Similarly, respondents who stated that cervical cancer is a severe disease (unadjusted $\mathrm{PR}=5.68$, 95\% CI $=2.89-11.12$ ), believed were at risk of getting cervical cancer (unadjusted PR $=1.72,95 \% \mathrm{CI}=1.42$ 2.07), stated that; cervical cancer screening is important (unadjusted $\mathrm{PR}=5.28,95 \% \mathrm{CI}=2.70-10.32$ ), the chances of curing cervical cancer are better when the disease is discovered at an early stage (unadjusted PR $=1.38$, 95\% $\mathrm{CI}=1.23-1.55)$, and that their family would approve of 
Table 2 Knowledge factors with intention to screen and willingness to vaccinate daughters against cervical cancer

\begin{tabular}{|c|c|c|c|c|c|c|}
\hline \multirow[b]{2}{*}{ Characteristic } & \multicolumn{3}{|c|}{ Intention to screen $(n=819)$} & \multicolumn{3}{|c|}{ Willingness to vaccinate daughters $(n=813)$} \\
\hline & N (\%) & Unadjusted PR (95\% Cl) & $p$-value & N (\%) & Unadjusted PR (95\% Cl) & $p$-value \\
\hline \multicolumn{7}{|c|}{ Early detection of cervical cancer is helpful } \\
\hline No & $36(80.0)$ & & & $39(86.7)$ & & \\
\hline Yes & $783(94.2)$ & $1.18[1.02-1.36]$ & $0.029^{*}$ & $774(93.6)$ & $1.08[0.96-1.21]$ & 0.194 \\
\hline \multicolumn{7}{|c|}{ Cervical cancer can be prevented } \\
\hline No & $246(92.8)$ & & & $246(92.1)$ & & \\
\hline Yes & $573(93.8)$ & $1.01[0.97-1.05]$ & 0.611 & $567(93.7)$ & $1.02[0.98-1.06]$ & 0.412 \\
\hline \multicolumn{7}{|c|}{ Knew recommended age for start of cervical cancer screening } \\
\hline No & $785(93.6)$ & & & $782(93.5)$ & & \\
\hline Yes & $34(91.9)$ & 0.98 [0.89-1.08] & 0.717 & $31(86.1)$ & $0.92[0.81-1.05]$ & 0.221 \\
\hline
\end{tabular}

Knew that one can be vaccinated against cervical cancer

\begin{tabular}{|c|c|c|}
\hline No & $282(90.7)$ & \\
\hline Yes & 537 (95.0) & $1.05[1.01-1.09]$ \\
\hline
\end{tabular}

Knew more than one preventive measure for cervical cancer

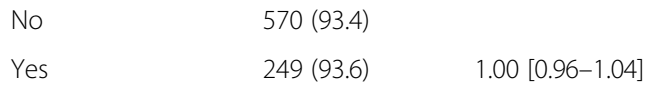

Knew more than one symptom of cervical cancer

$\begin{array}{lll}\text { No } & 382(95.0) & \\ \text { Yes } & 437(92.2) & 0.97[0.94-1.00]\end{array}$

Knew at least one test for cervical cancer

\begin{tabular}{|c|c|c|c|c|c|c|}
\hline No & $455(95.8)$ & & & $448(94.9)$ & & \\
\hline Yes & $364(90.8)$ & $0.95[0.91-0.98]$ & $0.004^{*}$ & $365(91.2)$ & $0.96[0.93-0.99]$ & $0.036^{*}$ \\
\hline \multicolumn{7}{|c|}{ Knew someone who had ever been screened for cervical cancer } \\
\hline No & $538(93.6)$ & & & $546(94.5)$ & & \\
\hline Yes & $281(93.4)$ & $0.99[0.96-1.03]$ & 0.905 & $267(90.8)$ & $0.96[0.92-1.00]$ & 0.062 \\
\hline \multicolumn{7}{|c|}{ Knew someone who had ever been diagnosed with cervical cancer } \\
\hline No & $569(93.3)$ & & & $576(93.9)$ & & \\
\hline Yes & $250(93.9)$ & $1.00[0.97-1.04]$ & 0.691 & $237(91.5)$ & $0.97[0.93-1.01]$ & 0.218 \\
\hline \multicolumn{7}{|c|}{ Knew someone who had died from cervical cancer } \\
\hline No & $568(93.3)$ & & & 572 (93.6) & & \\
\hline Yes & $251(94.0)$ & 1.00 [0.97-1.04] & 0.676 & 241 (92.3) & $0.99[0.95-1.03]$ & 0.507 \\
\hline
\end{tabular}

children being vaccinated against cervical cancer (unadjusted $\mathrm{PR}=6.38,95 \% \mathrm{CI}=3.49-11.63)$ had a significantly higher willingness to vaccinate their daughters against the disease (Table 4).

Health facility factors associated with intention to screen and willingness to vaccinate against cervical cancer

Respondents who accessed health care (unadjusted PR = $0.95,95 \% \mathrm{CI}=0.92-0.99)$ and reproductive health care (unadjusted $\mathrm{PR}=0.96,95 \% \mathrm{CI}=0.92-0.99$ ) from a government health facility, and those who knew where cervical cancer screening was provided (unadjusted $\mathrm{PR}=$ $0.94,95 \% \mathrm{CI}=0.91-0.98$ ) had a lower intention to screen for cervical cancer compared with their counterparts. On the other hand, respondents who lived within 5 kilometres from the health facility where screening services were provided had a higher intention to screen for cervical cancer (unadjusted PR $=1.12$, 95\% CI $=1.04-1.20$ ). Similarly, respondents who lived within 5 kilometres from the health facility had a higher willingness for vaccination of their daughters (unadjusted $P R=1.13,95 \% C I=1.05-1.21$ ). In contrast, there was less willingness for respondents who accessed health care (unadjusted $\mathrm{PR}=0.9695 \%$ $\mathrm{CI}=0.92-0.99$ ) and reproductive health care (unadjusted $\mathrm{PR}=0.95,95 \% \mathrm{CI}=0.91-0.98)$ from a government health facility, those who found it easy to access reproductive health care (unadjusted $\mathrm{PR}=0.97$, 95\% CI $=0.93-1.00)$, and those who knew where 
Table 4 Health facility factors with intention to screen and willingness to vaccinate daughters against cervical cancer

\begin{tabular}{|c|c|c|c|c|c|c|}
\hline \multirow[b]{2}{*}{ Characteristic } & \multicolumn{3}{|c|}{ Intention to screen $(n=819)$} & \multicolumn{3}{|c|}{ Willingness to vaccinate daughters $(n=813)$} \\
\hline & N (\%) & Unadjusted PR (95\% Cl) & $p$-value & $\mathrm{N}(\%)$ & Unadjusted PR (95\% Cl) & $p$-value \\
\hline \multicolumn{7}{|c|}{ Where health care is accessed when sick } \\
\hline Private facility & $166(97.1)$ & & & $168(96.5)$ & & \\
\hline Government facility & $653(92.6)$ & $0.95[0.92-0.99]$ & $0.006^{*}$ & $645(92.4)$ & $0.96[0.92-0.99]$ & $0.015^{*}$ \\
\hline \multicolumn{7}{|c|}{ Where reproductive health care is accessed } \\
\hline Private facility & $153(96.8)$ & & & $157(97.5)$ & & \\
\hline Government facility & $666(92.8)$ & $0.96[0.92-0.99]$ & $0.016^{*}$ & $656(92.3)$ & $0.95[0.91-0.98]$ & $0.001^{*}$ \\
\hline \multicolumn{7}{|c|}{ Ease of access of reproductive health care } \\
\hline Very/somewhat difficult & $372(92.8)$ & & & $378(94.9)$ & & \\
\hline Not difficult & $447(94.1)$ & $1.01[0.98-1.05]$ & 0.428 & $435(91.8)$ & $0.97[0.93-1.00]$ & 0.056 \\
\hline \multicolumn{7}{|c|}{ Have challenges in accessing reproductive health care } \\
\hline Yes & $480(92.5)$ & & & $487(94.0)$ & & \\
\hline No & $339(95.0)$ & $1.02[0.99-1.06]$ & 0.131 & $326(92.1)$ & $0.98[0.94-1.02]$ & 0.279 \\
\hline \multicolumn{7}{|c|}{ Ever been recommended for screening by health worker } \\
\hline No & $749(94.0)$ & & & $737(92.9)$ & & \\
\hline Yes & 70 (88.6) & $0.94[0.87-1.02]$ & 0.155 & $76(96.2)$ & $1.03[0.99-1.08]$ & 0.157 \\
\hline \multicolumn{7}{|c|}{ Knew where cervical cancer screening is provided } \\
\hline No & $496(95.7)$ & & & $489(95.9)$ & & \\
\hline Yes & $323(90.2)$ & $0.94[0.91-0.98]$ & $0.003^{*}$ & $324(89.5)$ & $0.93[0.89-0.97]$ & $0.001^{*}$ \\
\hline \multicolumn{7}{|c|}{ Distance to health facility where screening is done $(n-372)$} \\
\hline 5 or more $\mathrm{km}$ & $140(84.8)$ & & & $139(84.2)$ & & \\
\hline Less than $5 \mathrm{~km}$ & $183(94.8)$ & $1.12[1.04-1.20]$ & $0.003^{*}$ & $187(94.9)$ & $1.13[1.05-1.21]$ & $0.001^{*}$ \\
\hline \multicolumn{7}{|c|}{ Distance to nearest health facility } \\
\hline 5 or more $\mathrm{km}$ & $128(91.4)$ & & & $125(89.9)$ & & \\
\hline Less than $5 \mathrm{~km}$ & $691(93.9)$ & $1.03[0.97-1.08]$ & 0.336 & $688(93.9)$ & $1.04[0.98-1.10]$ & 0.153 \\
\hline
\end{tabular}

PR $=0.92$, 95\% CI: 0.85-0.98). Among attitude factors, although not statistically significant, respondents who stated that cervical cancer was a severe disease had an $81 \%$ higher intention to screen (adjusted PR $=1.81$ 95\% CI: 0.96-3.42) while the prevalence of intention to screen was $37 \%$ higher among respondents whose families would approve of children being vaccinated against cervical cancer (adjusted $\mathrm{PR}=1.37,95 \% \mathrm{CI}$ : 0.83-2.26) compared with their counterparts.

No sociodemographic characteristic was associated with willingness to vaccinate daughters against cervical cancer. Additionally, respondents who knew more than one symptom of cervical cancer had a lower prevalence of willingness to vaccinate daughters against cervical cancer (adjusted PR $=0.89,95 \%$ CI: 0.81-0.98). Although not statistically significant, the prevalence of willingness to vaccinate daughters was over 4 times higher among respondents who stated that cervical cancer screening was important (adjusted PR $=4.36,95 \%$ CI: 0.38-49.2) and over 3 times higher among those whose families would approve of children being vaccinated against cervical cancer (adjusted PR $=3.87,95 \%$ CI: 0.79-19.04) compared with their counterparts. No attitude and health facility factors were associated with willingness to vaccinate daughters against cervical cancer (Table 6).

\section{Discussion}

This study assessed the socio-demographic, knowledge, attitudinal and health facility factors associated with women's intention to screen, and willingness to vaccinate their daughters against cervical cancer in eastern Uganda. Overall, 91\% of respondents reported intention to screen for cervical cancer in the subsequent six months. This intention to screen increased to $92 \%$ if the service would be offered at an affordable cost, and to $95.9 \%$ if provided free of charge. This intention to screen for cervical cancer is higher than the $63 \%$ reported by a previous study conducted in southern Ugandan [18]. Although intention to screen was this high and respondents relatively knowledgeable about cervical cancer and screening [35], actual uptake of cervical cancer screening in this population of women was very low at $4.8 \%$ as 
Table 5 Socio-demographic characteristics with intention to screen, and willingness to vaccinate daughters against cervical cancer

\begin{tabular}{|c|c|c|c|c|c|c|}
\hline \multirow[t]{2}{*}{ Characteristic } & \multicolumn{2}{|c|}{ Intention to screen $(n=819)$} & \multirow[b]{2}{*}{$p$-value } & \multicolumn{3}{|c|}{ Willingness to vaccinate daughters $(n=813)$} \\
\hline & $\mathrm{N}(\%)$ & Unadjusted PR (95\% Cl) & & $\mathrm{N}(\%)$ & Unadjusted PR (95\% Cl) & $p$-value \\
\hline \multicolumn{7}{|l|}{ District } \\
\hline Bugiri & $388(89.0)$ & & & $404(90.9)$ & & \\
\hline Mayuge & $431(97.9)$ & $1.10[1.06-1.14]$ & $<0.001^{*}$ & $409(95.6)$ & $1.05[1.01-1.09]$ & $0.007^{*}$ \\
\hline \multicolumn{7}{|l|}{ Residence } \\
\hline Rural & $551(92.6)$ & & & $542(91.9)$ & & \\
\hline Semi-urban/urban & $268(95.4)$ & $1.03[0.99-1.06]$ & 0.093 & $271(96.1)$ & $1.05[1.01-1.08]$ & $0.009^{*}$ \\
\hline \multicolumn{7}{|l|}{ Age (years) } \\
\hline $25-39$ & $643(93.7)$ & & & $638(93.5)$ & & \\
\hline $40-49$ & $176(92.6)$ & $0.99[0.94-1.03]$ & 0.603 & $175(92.1)$ & $0.98[0.94-1.03]$ & 0.508 \\
\hline \multicolumn{7}{|l|}{ Education level } \\
\hline None/primary & $617(94.0)$ & & & $610(93.7)$ & & \\
\hline Post primary & $202(91.8)$ & $0.98[0.93-1.02]$ & 0.283 & $203(91.9)$ & $0.98[0.94-1.02]$ & 0.376 \\
\hline \multicolumn{7}{|l|}{ Religion } \\
\hline Muslims & $353(94.9)$ & & & $350(94.6)$ & & \\
\hline Christians & $466(92.5)$ & $0.97[0.94-1.00]$ & 0.138 & $463(92.2)$ & $0.97[0.94-1.01]$ & 0.159 \\
\hline \multicolumn{7}{|l|}{ Marital status } \\
\hline Single & $118(92.9)$ & & & $120(93.7)$ & & \\
\hline Married & $701(93.6)$ & $1.01[0.96-1.06]$ & 0.782 & $693(93.1)$ & $0.99[0.95-1.04]$ & 0.795 \\
\hline \multicolumn{7}{|l|}{ Occupation } \\
\hline Farming & $458(94.2)$ & & & $470(95.5)$ & & \\
\hline $\begin{array}{l}\text { Other (business, housewife, } \\
\text { civil servant) }\end{array}$ & $361(92.6)$ & $0.98[0.95-1.02]$ & 0.325 & $343(90.3)$ & $0.94[0.91-0.98]$ & $0.004^{*}$ \\
\hline \multicolumn{7}{|l|}{ Parity } \\
\hline Above four & $426(92.4)$ & & & $429(93.5)$ & & \\
\hline Four and below & $393(94.7)$ & $1.02[0.99-1.06]$ & 0.167 & $384(92.9)$ & $0.99[0.96-1.03]$ & 0.776 \\
\hline \multicolumn{7}{|l|}{ Household monthly income } \\
\hline Less than $\$ 40$ & $551(91.4)$ & & & $552(91.8)$ & & \\
\hline$\$ 40$ and above & $268(98.2)$ & $1.07[1.04-1.11]$ & $<0.001^{*}$ & $261(96.3)$ & $1.05[1.01-1.08]$ & 0.005 \\
\hline \multicolumn{7}{|l|}{ Household head } \\
\hline No & $688(93.3)$ & & & $688(93.3)$ & & \\
\hline Yes & $131(94.2)$ & $1.01[0.96-1.06]$ & 0.681 & $125(92.6)$ & $0.99[0.94-1.04]$ & 0.756 \\
\hline \multicolumn{7}{|l|}{ Ever tested for HIV } \\
\hline No & $133(94.3)$ & & & $133(95.0)$ & & \\
\hline Yes & $686(93.3)$ & $0.99[0.95-1.03]$ & 0.644 & $680(92.9)$ & $0.98[0.94-1.02]$ & 0.307 \\
\hline \multicolumn{7}{|c|}{ Ever used modern family planning method } \\
\hline No & $283(92.5)$ & & & $279(91.8)$ & & \\
\hline Yes & $536(94.0)$ & $1.02[0.98-1.06]$ & 0.392 & $534(94.0)$ & $1.02[0.98-1.06]$ & 0.233 \\
\hline \multicolumn{7}{|c|}{ Cervical cancer screening status } \\
\hline Not screened & $785(94.1)$ & & & $774(93.2)$ & & \\
\hline Screened & $34(80.9)$ & $0.86[0.74-0.99]$ & $0.046^{*}$ & $39(92.9)$ & $0.99[0.91-1.08]$ & 0.923 \\
\hline
\end{tabular}


Table 6 Independent predictors of intention to screen and willingness to vaccinate daughters against cervical cancer

\begin{tabular}{|c|c|c|c|c|}
\hline & Intention to screen & & Willingness to vaccina & \\
\hline Characteristic & Adjusted PR (95\% Cl) & $p$-value & Adjusted PR (95\% Cl) & $p$-value \\
\hline \multicolumn{5}{|l|}{ District (Bugiri) } \\
\hline Mayuge & $1.06[0.99-1.13]$ & 0.072 & $1.05[0.97-1.13]$ & 0.248 \\
\hline \multicolumn{5}{|l|}{ Residence (Rural) } \\
\hline Semi-urban/urban & $1.05[0.98-1.14]$ & 0.155 & - & - \\
\hline \multicolumn{5}{|l|}{ Age in years (25-39) } \\
\hline $40-49$ & $1.01[0.92-1.12]$ & 0.845 & $1.04[0.99-1.10]$ & 0.133 \\
\hline \multicolumn{5}{|c|}{ Education level (None/primary) } \\
\hline Post primary & $0.93[0.84-1.03]$ & 0.170 & $0.97[0.92-1.02]$ & 0.231 \\
\hline \multicolumn{5}{|l|}{ Religion (Muslims) } \\
\hline Christians & $0.92[0.85-1.00]$ & 0.059 & $0.97[0.93-1.01]$ & 0.172 \\
\hline \multicolumn{5}{|c|}{ Household monthly income (Less than $\$ 40$ ) } \\
\hline$\$ 40$ and above & $1.11[1.03-1.20]$ & $0.004^{*}$ & $1.03[0.99-1.07]$ & 0.149 \\
\hline \multicolumn{5}{|c|}{ Cervical cancer screening status (No) } \\
\hline Yes & $0.81[0.67-0.99]$ & $0.037^{*}$ & $1.01[0.93-1.11]$ & 0.769 \\
\hline
\end{tabular}

Knowledge factors

Early detection of cervical cancer is helpful (No)

$\begin{array}{lll}\text { Yes } & 1.07[0.92-1.24] & 0.407\end{array}$

Knew that one can be vaccinated against cervical cancer (No)

Yes $\quad 1.00[0.92-1.08]$

0.998

$0.99[0.96-1.04]$

0.959

Knew more than 1 preventive measure for cervical cancer (No)

Yes

$1.04[0.99-1.09]$

0.136

Knew more than 1 symptom of cervical cancer (No)

Yes

$0.93[0.86-1.01]$

0.078

$0.89[0.81-0.98]$

$0.020^{*}$

Knew at least one test for cervical cancer (No)

Yes

$0.92[0.85-0.98]$

$0.017^{*}$

$0.99[0.93-1.05]$

0.655

Attitude factors

Cervical cancer is a severe disease (No)

Yes

$1.81[0.96-3.42]$

0.068

$1.36[0.79-2.30]$

0.258

I am at risk of getting cervical cancer (No)

Yes

Cervical cancer screening is important (No)

Yes

$0.61[0.34-1.09]$

0.097

$4.36[0.38-49.2]$

0.234

Only women who are sexually active need cervical cancer screening (Yes)

No

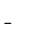

$1.02[0.97-1.06]$

0.479

Women who have had sexually transmitted diseases are more likely to get cervical cancer (No)

Yes $\quad 1.05[0.97-1.14] \quad 0.190$

$1.03[0.97-1.09]$

0.303

Chances of curing cervical cancer are better when the disease is discovered at an early stage (No)

Yes

$0.99[0.92-1.08] \quad 0.947$

$0.97[0.91-1.03]$

0.338

Cervical cancer is not a death sentence for most people (No)

Yes

$-$

$0.99[0.95-1.04]$

0.790

There is much a woman can do to reduce her chances of getting cervical cancer (No)

Yes

$1.02[0.95-1.08]$

0.697

$1.00[0.97-1.03]$

0.929

Women who have cervical cancer will have some symptoms showing it (Yes) 
Table 6 Independent predictors of intention to screen and willingness to vaccinate daughters against cervical cancer (Continued)

\begin{tabular}{|c|c|c|c|c|}
\hline No & $0.86[0.71-1.06]$ & 0.157 & $0.86[0.72-1.02]$ & 0.085 \\
\hline \multicolumn{5}{|c|}{ Cervical cancer runs in families (No) } \\
\hline Yes & - & - & $0.98[0.95-1.02]$ & 0.410 \\
\hline \multicolumn{5}{|c|}{ Women only need cervical cancer screening tests during child bearing years (No) } \\
\hline No & $0.99[0.92-1.06]$ & 0.802 & $0.97[0.93-1.02]$ & 0.228 \\
\hline \multicolumn{5}{|c|}{ Their family would approve of children being vaccinated against cervical cancer (No) } \\
\hline Yes & $1.37[0.83-2.26]$ & 0.217 & $3.87[0.79-19.04]$ & 0.096 \\
\hline \multicolumn{5}{|l|}{ Health facility factors } \\
\hline \multicolumn{5}{|c|}{ Where health care is accessed (Private facility) } \\
\hline Government facility & $0.93[0.82-1.05]$ & 0.240 & $1.03[0.87-1.20]$ & 0.749 \\
\hline \multicolumn{5}{|c|}{ Where reproductive health care is accessed (Private facility) } \\
\hline Government facility & $1.11[0.89-1.39]$ & 0.351 & $0.95[0.86-1.05]$ & 0.347 \\
\hline \multicolumn{5}{|c|}{ Knew where cervical cancer screening is provided (No) } \\
\hline Yes & $1.13[0.97-1.32]$ & 0.121 & $1.08[0.96-1.23]$ & 0.189 \\
\hline \multicolumn{5}{|c|}{ Distance to health facility where screening is done (5 or more $\mathrm{km}$ ) } \\
\hline Less than $5 \mathrm{~km}$ & $1.07[0.98-1.17]$ & 0.124 & $1.03[0.98-1.08]$ & 0.271 \\
\hline
\end{tabular}

reported in a related study [19]. This therefore calls for an in-depth exploration of the barriers that could be limiting most of these women from accessing cervical cancer screening. In fact, respondents who did not intend to screen majorly stated that screening was neither necessary nor important highlighting the presence of knowledge gaps that should be filled through adequate sensitisation of women to appreciate the severity of the disease and increase their risk perception. Therefore, increasing access to cervical cancer screening services and further education of women is expected to increase uptake rates of screening services which are currently low in developing countries including Uganda.

The target group for HPV vaccinations being prepubescent girls, parental approval is required for the success of vaccination programmes [24]. In this study, we found a high willingness of $90.4 \%$ among women to vaccinate their daughters against cervical cancer. This is in line with proportions of over $90 \%$ reported by Kenyan [36, 37] and Ghanaian [7] studies. However, these studies majorly targeted urban women at health centres unlike our study that had majority rural participants in the community. This high willingness in our study could have been contributed to by the high acceptability and uptake of vaccines in Uganda [5], a determinant of HPV vaccine acceptability among parents [7, 25, 28]. There are also ongoing campaigns by the government of Uganda aimed at HPV vaccinations of girls aged between 9 to 13 years which could have increased awareness of cervical cancer and vaccinations. As there is high willingness to vaccinate daughters, the focus of various stakeholders such as the Ministry of Health and
Non-governmental Organisations should be turning this into actual practice through increasing service availability and accessibility by using effective strategies such as schools, health centres, and community based programmes $[5,8]$.

In the study, there was a significant association between having higher income and intention to screen against cervical cancer. Moreover, intention to screen increased with possibilities of affordable and free cervical cancer screening which could highlight the cost of the procedure as a barrier as reported elsewhere [16, 19, 38]. Prior studies have also found associations between higher income and uptake of cervical cancer screening $[17,39,40]$. This highlights the need to ensure the provision of affordable or free cervical cancer screening services to increase uptake of the service among women in developing countries. We also found that respondents who had already accessed screening, and those who knew at least one test for cervical cancer were less likely to intend to screen in the subsequent six months. It is likely that respondents who knew the screening tests had been screened for cervical cancer as earlier highlighted [19]. Since these respondents had already been screened, though at various intervals [19], they could have been reluctant to access screening in the following six months. Indeed, the WHO recommends screening for cervical cancer at 3 year intervals among women aged 30 years and above [11]. Similarly, the Ugandan Ministry of Health strategic plan for cervical cancer prevention and control 2010-2014 recommends screening for cervical cancer once every three years for HIV negative women, and annually for those who are 
HIV positive [6]. Nonetheless, a study in Kenya [17] found lower intentions to screen among women who had already been screened while a Chinese study reported otherwise [41]. Respondents who knew more than one symptom of the disease had lower willingness to vaccinate their daughters. It is possible that such respondents were reluctant to take up interventions in cases where they knew how the disease could manifest yet they had not observed these. Indeed, it has been reported that women may for example not access cervical cancer screening because they do not have any signs and symptoms of the disease $[18,19]$. Sensitization campaigns should emphasize the asymptomatic nature of cervical cancer especially during its early stages and encourage women to access cervical cancer services even when they do not have any signs or symptoms of the disease. Among the attitudinal factors, although not statistically significant, respondents whose families would approve of daughters being vaccinated against cervical cancer were more likely to intend to screen for the disease, and more willing to have their daughters vaccinated against it. Study respondents being women, this could indicate a gender influence which has been shown to affect health care decision making in many African households [42, 43]. For example, previous studies have shown that sometimes women may require approval and financial support from their husbands to seek cervical cancer screening $[18,42,43]$ emphasizing the importance of social influence from important others such as spouses. On the other hand, this finding could indicate the desire for collective decision making among parents especially when it comes to the vaccination of their daughters as reported by another study [7]. Overall, this underscores the need for involvement of all societal groups especially men in programmes aimed at increasing cervical cancer screening and vaccination.

This study examined intention to screen, and willingness to vaccinate daughters among a majorly rural population, and had a large sample size compared with other sub-Saharan Africa studies. However, some elements of this study for example intention to screen, and willingness to vaccinate daughters could have been affected by the tendency for study participants to give socially desirable responses which could have introduced bias. We also did not assess knowledge about HPV and the vaccine whose relationship with vaccination acceptability would have been relevant to explore, and neither did we assess reasons for willingness to vaccinate daughters among the sampled women. Nevertheless, this study contributes important information regarding intention to screen among women and willingness to vaccinate daughters against cervical cancer and associated factors which can inform future studies as well as cervical cancer programmes.

\section{Conclusion}

There was a very high intention to screen and willingness to vaccinate daughters against cervical cancer among women in eastern Uganda. Only higher income predicted intention to screen for cervical cancer while respondents whose families would approve of their daughters being vaccinated against cervical cancer were more likely to intend to screen for the disease and willing for their daughters to be vaccinated. To take advantage of the high rates of intention to screen, and willingness to vaccinate daughters against cervical cancer, opportunities should be availed for women to access screening, and for girls to be vaccinated against the disease. In addition, the involvement of all societal groups especially men in programmes aimed at increasing screening and vaccinations of girls could increase uptake of these services.

\section{Abbreviations}

HIV: Human immunodeficiency virus; HPV: Human papilloma virus; PR: Prevalence ratio; WHO: World Health Organization

\section{Acknowledgements}

We thank the study participants and research assistants for taking part in the study.

\section{Funding}

This work was supported by Training Health Researchers into Vocational Excellence in East Africa (THRiVE), grant number 087540 funded by the Wellcome Trust. Its contents are solely the responsibility of the authors and do not necessarily represent the official views of the supporting offices. The funders had no role in study design, data collection and analysis, decision to publish, or preparation of the manuscript.

\section{Availability of data and materials}

Due to ethical restrictions related to protecting patient privacy, data cannot be made publicly available. Data are available upon request from the corresponding author

\section{Authors' contributions}

RN conceived the study, contributed to its design, data collection and analysis, and drafted the manuscript. TM and DM contributed in designing the study, data collection and analysis, and critical review of the manuscript. GM, AAH and JS contributed to the data analysis and critical review of the manuscript. All authors read and approved the final manuscript.

\section{Competing interests}

The authors have no competing interests to declare.

\section{Consent for publication}

Not applicable.

\section{Ethics approval and consent to participate}

This study received ethical approval from Makerere University School of Public Health Higher Degrees, Research and Ethics Committee, and was registered by the Uganda National Council for Science and Technology. Permission to carry out the study was further obtained from the local administration of Bugiri and Mayuge districts. Participation in the study was voluntary and respondents provided written informed consent only after being explained to the details of the study including the benefits and potential risks of participation.

\section{Publisher's Note}

Springer Nature remains neutral with regard to jurisdictional claims in published maps and institutional affiliations. 


\section{Received: 27 September 2016 Accepted: 8 March 2017} Published online: 14 March 2017

\section{References}

1. Bingham A, Bishop A, Coffey P, Winkler J, Bradley J, Dzuba I, Agurto I. Factors affecting utilization of cervical cancer prevention services in lowresource settings. Salud Publica Mex. 2003;45 Suppl 3:S408-416.

2. Herdman C, Sherris J. Planning appropriate cervical cancer prevention programs. 2nd ed. Seattle: Program for Appropriate Technology in Health (PATH); 2000. http://screening.iarc.fr/doc/cxca-planning-appro-prog-guide.pdf

3. Gakidou E, Nordhagen S, Obermeyer Z. Coverage of cervical cancer screening in 57 countries: low average levels and large inequalities. PLoS Med. 2008;5(6):e132.

4. Holowaty P, Miller AB, Rohan T, To T. Natural history of dysplasia of the uterine cervix. J Natl Cancer Inst. 1999;91(3):252-8.

5. Program for Appropriate Technology in Health (PATH), Child Health and Development Centre (CHDC), the Uganda National Expanded Program on Immunization (UNEPI). HPV vaccination in Africa: Lessons learned from a pilot program in Uganda. Seattle: PATH; 2011. https://www.path.org/ publications/files/RH_hpv_lessons_learned_uganda.pdf.

6. Ministry of Health. Strategic plan for cervical cancer prevention and control in Uganda 2010-2014. Kampala, Uganda; 2010. http://www.rho.org/files/ PATH_Uganda_cxca_strat_plan_2010-2014.pdf

7. Coleman MA, Levison J, Sangi-Haghpeykar H. HPV vaccine acceptability in Ghana, West Africa. Vaccine. 2011;29(23):3945-50.

8. LaMontagne DS, Barge S, Le Thi N, Mugisha E, Penny ME, Gandhi S, Janmohamed A, Kumakech E, Mosqueira NR, Nguyen NQ. Human papillomavirus vaccine delivery strategies that achieved high coverage in low-and middleincome countries. Bull World Health Organ. 2011;89(11):821-30.

9. Sankaranarayanan R, Esmy PO, Rajkumar R, Muwonge R, Swaminathan R, Shanthakumari S, Fayette J-M, Cherian J. Effect of visual screening on cervical cancer incidence and mortality in Tamil Nadu, India: a clusterrandomised trial. Lancet. 2007;370(9585):398-406.

10. American Cancer Society. Cancer facts and figures 2010. Atlanta: American Cancer Society; 2010. https://old.cancer.org/acs/groups/content/@nho/ documents/document/acspc-024113.pdf.

11. World Health Organization: Comprehensive cervical cancer control: a guide to essential practice. Geneva: World Health Organization; 2006.

12. Wellensiek N, Moodley M, Moodley J, Nkwanyana N. Knowledge of cervical cancer screening and use of cervical screening facilities among women from various socioeconomic backgrounds in Durban, Kwazulu Natal, South Africa. Int J Gynecol Cancer. 2002;12(4):376-82.

13. Ayinde $\mathrm{O}$, Omigbodun A. Knowledge, attitude and practices related to prevention of cancer of the cervix among female health workers in Ibadan. J Obstet Gynaecol. 2003;23(1):59-62.

14. Gichangi P, Estambale B, Bwayo J, Rogo K, Ojwang S, Opiyo A, Temmerman M. Knowledge and practice about cervical cancer and Pap smear testing among patients at Kenyatta National Hospital, Nairobi, Kenya. Int J Gynecol Cancer. 2003;13(6):827-33.

15. Gharoro E, Ikeanyi E. An Appraisal of The Level of Awareness and Utilization of The Pap Smear as A Cervical Cancer Screening Test Among Female Health Workers In A Tertiary Health Institution. Int J Gynecol Cancer. 2006;16(3):1063-8.

16. Were E, Nyaberi Z, Buziba N. Perceptions of risk and barriers to cervica cancer screening at Moi Teaching and Referral Hospital (MTRH), Eldoret. Kenya Afr Health Sci. 2011;11(1):58-64.

17. Sudenga SL, Rositch AF, Otieno WA, Smith JS. Knowledge, attitudes, practices, and perceived risk of cervical cancer among Kenyan women: brief report. Int J Gynecol Cancer. 2013;23(5):895-9.

18. Twinomujuni C, Nuwaha F, Babirye JN. Understanding the Low Level of Cervical Cancer Screening in Masaka Uganda Using the ASE Model: A Community-Based Survey. PLoS One. 2015;10(6):e0128498.

19. Ndejjo R, Mukama T, Musabyimana A, Musoke D. Uptake of Cervical Cancer Screening and Associated Factors among Women in Rural Uganda: A Cross Sectional Study. PLoS One. 2016;11(2):e0149696.

20. Bruni L B-RL, Serrano B, Brotons M, Cosano R, Muñoz J, Bosch FX, de Sanjosé S, Castellsagué X. Human Papillomavirus and Related Diseases in World. In: Summary Report. Barcelona: ICO Information Centre on HPV and Cancer (HPV Information Centre); 2014. http://www.hpvcentre.net/statistics/reports/XWX.pdf.

21. Bosch FX, Manos MM, Muñoz N, Sherman M, Jansen AM, Peto J, Schiffman MH, Moreno V, Kurman R, Shan KV. Prevalence of human papillomavirus in cervical cancer: a worldwide perspective. J Natl Cancer Inst. 1995;87(11):796-802.
22. Munoz N, Castellsagué X, de González AB, Gissmann L. HPV in the etiology of human cancer. Vaccine. 2006:24:S1-S10.

23. World Health Organization. Human papillomavirus vaccines: WHO position paper, October 2014-Recommendations. Vaccine. 2015;33(36): 4383-4384.

24. Zimet GD. Improving adolescent health: focus on HPV vaccine acceptance. Adolesc Health. 2005;37(6):S17-23.

25. Lenselink CH, Gerrits MM, Melchers WJ, Massuger LF, van Hamont D, Bekkers RL. Parental acceptance of human papillomavirus vaccines. Eur J Obstet Gynecol Reprod Biol. 2008;137(1):103-7.

26. Wani JA, Murokora D. Acceptability of HPV vaccine among young adolescent girls in Uganda: Young people's perspectives count. Int J Child and Adolesc Health. 2013;6(2):211.

27. Mouallif M, Bowyer HL, Festali S, Albert A, Filali-Zegzouti Y, Guenin S, Delvenne P. Waller J, Ennaji MM. Cervical cancer and HPV: awareness and vaccine acceptability among parents in Morocco. Vaccine. 2014;32(3):409-16.

28. Davis K, Dickman ED, Ferris D, Dias JK. Human papillomavirus vaccine acceptability among parents of 10-to 15-year-old adolescents. J Low Genit Tract Dis. 2004;8(3):188-94.

29. Kester LM, Zimet GD, Fortenberry JD, Kahn JA, Shew ML. A national study of HPV vaccination of adolescent girls: rates, predictors, and reasons for nonvaccination. Matern Child Health J. 2013;17(5):879-85.

30. Uganda Bureau of Statistics. The National Population and Housing Census 2014 - Main Report. Kampala, Uganda; 2014. http://www.ubos.org/ onlinefiles/uploads/ubos/NPHC/ NPHC\%202014\%20FINAL\%20RESULTS\%20REPORT.pdf.

31. Kish L. Survey sampling. 1965.

32. Barros AJ, Hirakata VN. Alternatives for logistic regression in cross-sectional studies: an empirical comparison of models that directly estimate the prevalence ratio. BMC Med Res Methodol. 2003;3(1):21.

33. Newcombe RG. A deficiency of the odds ratio as a measure of effect size. Stat Med. 2006;25(24):4235-40.

34 Schmidt $\mathrm{CO}$, Kohlmann T. When to use the odds ratio or the relative risk? Int J Public Health. 2008;53(3):165-7.

35. Mukama T, Ndejio R, Musabyimana A, Halage AA, Musoke D. Women's knowledge and attitudes towards cervical cancer prevention: a cross sectional study in Eastern Uganda. BMC Women's Health. 2017:17(1):9.

36. Rositch AF, Gatuguta A, Choi RY, Guthrie BL, Mackelprang RD, Bosire R, Manyara L, Kiarie JN, Smith JS, Farquhar C. Knowledge and acceptability of pap smears, self-sampling and HPV vaccination among adult women in Kenya. PLoS One. 2012;7(7):e40766.

37. Becker-Dreps S, Otieno WA, Brewer NT, Agot K, Smith JS. HPV vaccine acceptability among Kenyan women. Vaccine. 2010;28(31):4864-7.

38. Cunningham MS, Skrastins E, Fitzpatrick $R$, Jindal $P$, Oneko $O$, Yeates $K$ Booth CM, Carpenter J, Aronson KJ. Cervical cancer screening and HPV vaccine acceptability among rural and urban women in Kilimanjaro Region, Tanzania. BMJ Open. 2015;5(3):e005828

39. Mingo AM, Panozzo CA, DiAngi YT, Smith JS, Steenhoff AP, RamogolaMasire D, Brewer NT. Cervical cancer awareness and screening in Botswana. Int J Gynecol Cancer. 2012;22(4):638.

40. Ortiz AP, Hebl S, Serrano R, Fernandez ME, Suárez E, Tortolero-Luna G. Factors associated with cervical cancer screening in Puerto Rico. Preventing chronic disease. 2010;7(3):A58.

41. Gu C, Chan CW, He G-P, Choi K, Yang S-B. Chinese women's motivation to receive future screening: the role of social-demographic factors, knowledge and risk perception of cervical cancer. Eur J Oncol Nurs. 2013;17(2):154-61.

42. Mutyaba T, Faxelid E, Mirembe F, Weiderpass $E$. Influences on uptake of reproductive health services in Nsangi community of Uganda and their implications for cervical cancer screening. Reprod Health. 2007:4:4.

43. Lyimo FS, Beran TN. Demographic, knowledge, attitudinal, and accessibility factors associated with uptake of cervical cancer screening among women in a rural district of Tanzania: three public policy implications. BMC Public Health. 2012;12(1):22. 